\title{
Glycerol as high-permittivity liquid filler in dielectric silicone elastomers
}

\author{
Mazurek, Piotr Stanislaw; Yu, Liyun; Gerhard, R.; Wirges, W.; Skov, Anne Ladegaard
}

Published in:

Journal of Applied Polymer Science

Link to article, DOI:

10.1002/app.44153

Publication date:

2016

Document Version

Peer reviewed version

Link back to DTU Orbit

Citation (APA):

Mazurek, P. S., Yu, L., Gerhard, R., Wirges, W., \& Skov, A. L. (2016). Glycerol as high-permittivity liquid filler in dielectric silicone elastomers. Journal of Applied Polymer Science, 133(43), [44153].

https://doi.org/10.1002/app.44153

\section{General rights}

Copyright and moral rights for the publications made accessible in the public portal are retained by the authors and/or other copyright owners and it is a condition of accessing publications that users recognise and abide by the legal requirements associated with these rights.

- Users may download and print one copy of any publication from the public portal for the purpose of private study or research.

- You may not further distribute the material or use it for any profit-making activity or commercial gain

- You may freely distribute the URL identifying the publication in the public portal

If you believe that this document breaches copyright please contact us providing details, and we will remove access to the work immediately and investigate your claim. 


\title{
Glycerol as high-permittivity liquid filler in dielectric silicone elastomers
}

\author{
P. Mazurek, ${ }^{a}$ L. Yu, ${ }^{a}$ R. Gerhard, ${ }^{\mathrm{b}}$ W. Wirges, ${ }^{\mathrm{b}}$ A.L. Skov ${ }^{a} *$
}

${ }^{a}$ Danish Polymer Centre, Department of Chemical and Biochemical Engineering, Building 227, Technical University of Denmark, DK-2800 Kgs. Lyngby.

${ }^{b}$ Applied Condensed-Matter Physics, Institute of Physics and Astronomy, Faculty of Science, University of Potsdam, Karl-Liebknecht-Str. 24-25, 14476 Potsdam-Golm, Germany

\begin{abstract}
A recently reported novel class of elastomers was tested with respect to its dielectric properties. The new elastomer material is based on a commercially available polydimethylsiloxane (PDMS) composition, which has been modified by embedding glycerol droplets into its matrix. The approach has two major advantages that make the material useful in a dielectric actuator. First, the glycerol droplets efficiently enhance the dielectric constant which can reach astonishingly high values in the composite. Second, the liquid filler also acts as a softener that effectively decreases the elastic modulus of the composite. In combination with very low cost and easy preparation, the two property enhancements lead to an extremely attractive dielectric elastomer material. Experimental permittivity data are compared to various theoretical models that predict relative-permittivity changes as a function of filler loading, and the applicability of the models is discussed.
\end{abstract}



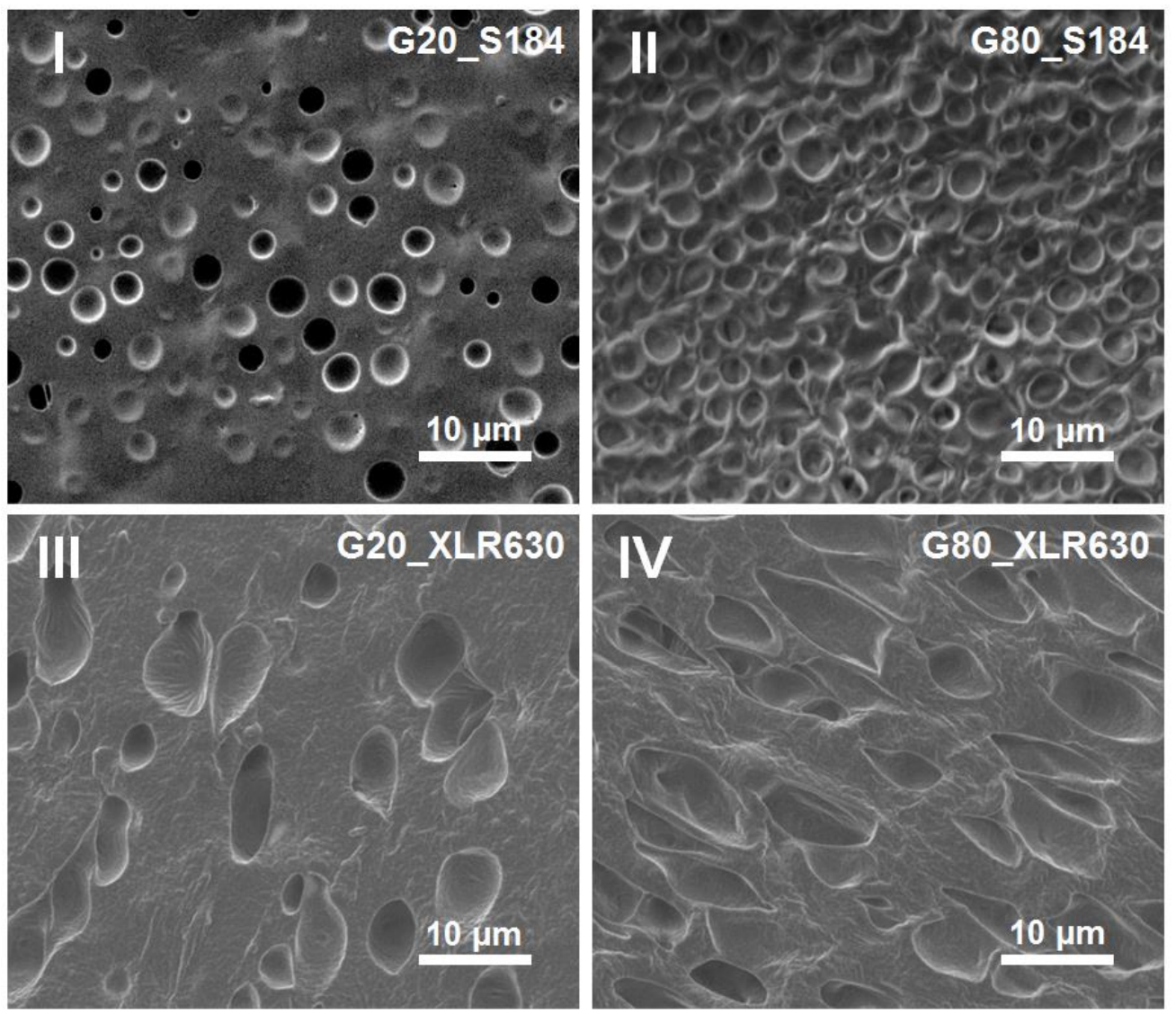


\section{Introduction}

The concept of electromechanical transduction in polymeric materials was investigated by Wilhelm Conrad Röntgen already in 1880 in a simple experiment where a rubber band was actuated by sprayed-on charges that caused electrostatic forces across its thickness. ${ }^{[1,2]}$ The early study became an inspiration for the research conducted nowadays on electroactive dielectric polymers, which intensified after 1990. Ever since, material scientists have worked on optimizing material properties and actuation conditions. ${ }^{[3-5]}$

It has been established that actuator performance strain in thickness direction $\left(\mathrm{s}_{\mathrm{z}}\right)$ depends on applied voltage (U), Young's modulus (Y) and initial thickness (d) of the elastomeric membrane and last, but not least, on the relative dielectric permittivity of the material $(\varepsilon)$, as shown in Equation (1). ${ }^{[6]} \varepsilon_{0}$ is the vacuum permittivity.

$$
s_{z}=\frac{\varepsilon_{0} \varepsilon}{Y}\left(\frac{U}{d}\right)^{2}
$$

Because of currently over-expensive power supply units, the risks of elastomer electrical breakdown and a risk of exceeding the space-charge threshold (resulting in accelerated electrical ageing), the applied voltage has to be kept at the lowest possible level. ${ }^{[7]}$ Young's modulus and elastomer thickness are materials properties that have to be adjusted for the respective application. For an actuator or a generator, a dielectric polymer with the highest possible dielectric permittivity should be the best choice. Promising examples of such materials are polyurethanes ${ }^{[8]}$ and poly(propylene oxide) ${ }^{[9]}$ with dielectric constants of 6.2 and 5.6 at $1 \mathrm{kHz}$, respectively. In both cases however the materials suffer from relatively low breakdown strength and a short lifetime. For this reason, attention has shifted towards very soft, high-dielectric constant polyacrylates and soft, but low-dielectric-constant PDMS. The most widely applied representatives of the polyacrylates, the 
commercial Very High Bond (VHB) Tapes from 3M, were recently reported to exhibit an impressively high power density of $280 \mathrm{~W} / \mathrm{kg}^{[10]}$ Yet the prepared generator was capable of withstanding less than 10 cycles, after which the energy generation drastically decreased. Therefore, McKay et al. asked whether it make sense to conduct research on efficient, but mechanically unstable polyacrylates. ${ }^{[11]}$ They suggested that investing time in a PDMS with lower energy density, but much higher durability would contribute more significantly to the development of dielectric elastomers.

PDMS has over the years developed into a key candidate for dielectric actuators and generators due to its long lifetime, high durability, low viscous losses, high breakdown strength, etc. Nevertheless, it suffers from a rather low dielectric constant, which directly influences the performance of actuators or generators. There have been a number of publications in which the dielectric constant of PDMS was successfully enhanced through simply blending a PDMS prepolymer with high-permittivity fillers. This method, despite being the easiest and most intuitive, offers a broad range of possibilities due to an essentially unlimited selection of fillers, amongst which one can find magnesium niobate-lead titanate, ${ }^{[12]}$ titanium dioxide, ${ }^{[13]}$ graphite, ${ }^{[14]}$ carbon nanotubes, ${ }^{[15]}$ graphene, ${ }^{[16]}$ conductive polymers, ${ }^{[17]}$ etc. A more sophisticated method of increasing the permittivity has been presented by Madsen et al., who covalently linked high-dielectric constant dipolar molecules to the elastomer backbone via a novel type of chain extender. ${ }^{[18,19]}$ Nevertheless, none of the reported elastomers presently meets the requirements that are foreseen for a highly efficient, reliable and thus also commercially attractive dielectric elastomer. The indisputable need for materials improvement leads to many unconventional approaches for reaching a breakthrough in the development of dielectric elastomer actuators. ${ }^{[20]}$

In our recent paper high-dielectric-constant liquids were suggested as fillers that are potentially capable of enhancing a material's dielectric constant. ${ }^{[21,22]}$ It was reported that water $(\varepsilon=80)$ 
incorporated and uniformly distributed within hydrophobic PDMS in the form of encapsulated discrete water droplets is capable of enhancing the dielectric constant of a given elastomer. On the other hand, the resulting composite material suffered from too high film thickness, which greatly limited its range of applications. Furthermore, water, as a volatile substance, was gradually evaporating from the composite, which was additionally facilitated by the substantial gas permeability of PDMS. ${ }^{[23]}$ Nevertheless, this approach, although having some drawbacks, proved our concept of polar liquids as useful high-permittivity fillers. The idea of using high-permittivity polar liquids as fillers in dielectric elastomers was also supported in a concept of generating water marbles that are responsive to high electric and magnetic fields. ${ }^{[24-27]}$ Liquid marbles are often described as non-stick droplets coated with micrometer-sized hydrophobic particles. ${ }^{[28]}$ The liquid marbles were proven to actuate upon exposure to sufficiently high electric fields. Due to dipole reorientation, water marbles change shape with the direction of an applied electric field. It has been postulated that the efficiency of actuation is directly related to the dielectric constant of the respective liquid. ${ }^{[2]}$ That postulate let us to hypothesize, that liquids might improve a dielectric elastomer actuation performance, provided that the liquid is non-volatile and is incorporated into the elastomer in the form of discrete droplets.

Here, our recently developed silicone elastomer composite was investigated with respect to its possible use as an electroactive dielectric polymer. A cross-section of the material is schematically presented in Figure 1. A detailed description of its preparation, as well as some relevant parameters and features, e.g. mechanical properties, can be found in our previous publication. ${ }^{[30]}$ Now the glycerol-containing PDMS is tested in terms of the dielectric properties at high and at low voltages. The approach offers a two-fold advantage from incorporating a high-permittivity liquid into a PDMS elastomer. First, the dielectric constant is significantly increased. Second, as shown in our previous paper ${ }^{[29]}$, zones of zero-stress are introduced by embedding discrete liquid droplets into the 
elastomer matrix. This leads to an overall decrease of Young's modulus in the composite, which is expected to enhance its actuation performance.

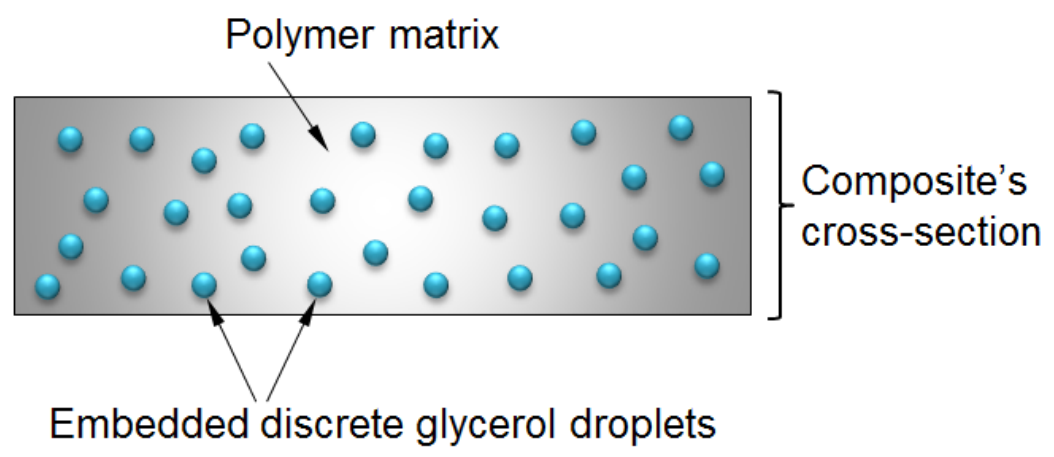

Figure 1. Schema of a glycerol-PDMS composite. Glycerol is embedded into a dielectric elastomer in the form of discrete droplets in order to avoid the formation of conductive pathways across the material.

\section{Experimental}

\subsection{Materials}

The two component Sylgard 184 (abbreviated as S184) silicone kit and the volatile methylsiloxane fluid OS-20 (abbreviated as OS20) were purchased from Dow Corning US. Powersil XLR 630 A/B (abbreviated as XLR630) was obtained from Wacker Chemie AG. Both silicones are platinum-catalysed hydrosilylation addition-cure systems reinforced with silica particles. A food-grade, glycerol was kindly provided by Emmelev A/S Denmark and was used as received. Tween 20, a polysorbate-based surfactant was obtained from Sigma Aldrich. Two different methyl-terminated poly(dimethylsiloxane-b-ethylene oxide) surfactant-like diblock copolymers were purchased from Polysciences Inc. where the lower-molecular-weight PDMS-PEG copolymer $(\mathrm{M}=600 \mathrm{~g} / \mathrm{mol})$ and the higher-molecular-weight PDMS-PEG copolymer $(\mathrm{M}=3000$ $\mathrm{g} / \mathrm{mol}$ ) had estimated molar ratios of PEG to PDMS blocks of 25:75 and 20:80, respectively. The 
PEG blocks were estimated to have a molecular weight of $\mathrm{M}=400 \mathrm{~g} / \mathrm{mol}$. The copolymer specifications were provided by the supplier. All chemicals were used as received with no further purification.

\subsection{Sample preparation}

Table 1. Glycerol - S184 formulations with corresponding volume fractions. ${ }^{\mathrm{a}}$

\begin{tabular}{|l|c|c|}
\hline Sample name & $\begin{array}{c}\text { Amount of } \\
\text { glycerol [phr] }\end{array}$ & $\begin{array}{c}\text { Volume fraction } \\
\text { of glycerol in } \\
\text { the sample }\end{array}$ \\
\hline G0_S184 & 0 & 0 \\
\hline G10_S184 & 10 & 0.075 \\
\hline G20_S184 & 20 & 0.141 \\
\hline G40_S184 & 40 & 0.246 \\
\hline G60_S184 & 60 & 0.329 \\
\hline G80_S184 & 80 & 0.395 \\
\hline G100_S184 & 100 & 0.450 \\
\hline G120_S184 & 120 & 0.495 \\
\hline
\end{tabular}

a Volume fractions for glycerol-XLR630 formulations are slightly different due to higher density of XLR630.

${ }^{\mathrm{b}}$ Sample names were formed using the pattern: GX_Y_Z, where G and X denote glycerol and the amount of glycerol phr added to a PDMS prepolymer, respectively, Y corresponds to the PDMS composition employed, and $\mathrm{Z}$ indicates supplementary components (in the approach discussed below, it corresponds to the thinning fluid OS-20).

c The abbreviation 'phr' used for describing glycerol content in all compositions corresponds to glycerol amount by weight per hundred weight parts of silicone rubber.

All composite prepolymer compositions were prepared via mixing all compounds with a dual asymmetric centrifuge SpeedMixer DAC 150 FVZ-K. The S184 was mixed in a ratio of $10: 1$ as recommended by the supplier. Subsequently, glycerol was added to the prepolymer, followed by 
vigorous stirring with the speed-mixer at 3500 r.p.m. for 5 minutes. The glycerol-PDMS prepolymer emulsion was cast onto a metal mold with $1 \mathrm{~mm}$ thick spacer and cured for $1 \mathrm{~h}$ at $80{ }^{\circ} \mathrm{C}$. Afterwards, the samples were kept at room temperature for at least 2 days prior to the measurements in order to ensure that eventual post-curing was completed. Due to very low vapor pressure an eventual evaporation of glycerol from the produced films is neglected. The amounts of incorporated glycerol by mass and the corresponding volume fractions are given in Table $\mathbf{1}$.

The Powersil XLR 630 was mixed in a ratio of $1: 1$ by weight, as recommended by the manufacturer, which was followed by the addition of glycerol. Subsequently $40 \mathrm{phr}$ of OS-20 fluid was added to the system. This low-molecular weight, low-viscosity, volatile and PDMS prepolymer-miscible substance effectively decreases viscosity of silicone compositions making them much easier to process. The speed-mixed compositions (3500 r.p.m. for 5 minutes, were cast onto a metal mold as in the previous case. Here again, the samples were cured for $1 \mathrm{~h}$ at $80{ }^{\circ} \mathrm{C}$. Subsequently, the samples were kept at room temperature for at least 7 days in order to ensure complete cross-linking of the material as well as evaporation of the remaining OS-20.

Samples with addition of a surfactant were prepared in the same way as the glycerol-S184 composites, adding the desired amounts of surfactant before speed-mixing.

\subsection{Methods}

Field Electron and Ion Co. (FEI) Inspect S Scanning Electron Microscope (SEM) was used to obtain images of typical cross-sections of fully crosslinked samples. A Novocontrol broadband dielectric spectrometer was employed to determine the real and the imaginary parts of the complex permittivities as well as the conductivities on the composite specimens. Disc-shaped samples with a diameter of $20 \mathrm{~mm}$ of diameter and a thickness of around $1 \mathrm{~mm}$ were subjected to an AC voltage of

$1 \mathrm{~V}$ amplitude in the frequency range between $10^{-1}$ and $10^{6} \mathrm{~Hz}$. A broadband dielectric 
spectrometer Novocontrol Alpha-A, equipped with a high-voltage booster HVB1000, was used to investigate the dielectric response of samples as a function of the applied voltage. Specimens were tested in a frequency range between $10^{-1}$ and $10^{-4} \mathrm{~Hz}$ (a narrower frequency range than in the case of the $1 \mathrm{~V}$ AC measurements, due to technical limitations). When discussing the resulting dielectric permittivities of the composites, the values at $1 \mathrm{kHz}$ are used.

\section{Results and discussion}

\subsection{SEM analysis of glycerol-PDMS composites}

Cross-sections of cross-linked composites were investigated by scanning electron microscopy (SEM). Images I and II, presented in Figure 2, correspond to cross-sections of samples G20_S184 and G80_S184, respectively. As can be seen, droplet concentration becomes higher with increasing amount of glycerol. However, the average droplet size remains almost unchanged. Furthermore, it was observed that when increasing the glycerol/PDMS ratio, a threshold concentration is finally reached (G130_S184), above which a droplet-like morphology is no longer present in the cross-linked material. As discussed previously, a network of interconnected glycerol channels is obtained instead, which leads to higher-conductivity paths in the material. ${ }^{[30]}$ Therefore, samples with more than $120 \mathrm{phr}$ of glycerol are not discussed here.

The addition of the thinning agent to XLR630 was shown to allow for the incorporation of significant amounts of glycerol (up to $120 \mathrm{phr}$ ) into the PDMS matrix and also facilitated processability. Without the addition of the diluting OS-20, a maximum possible glycerol loading of $40 \mathrm{phr}$ was observed. Above this limit, the glycerol-XLR630 prepolymer emulsions had a very broad size distribution of glycerol droplets, and the mixtures were phase-separated even after 
extended mixing. As depicted in Figure 2 (images III-IV), the morphology of spherical droplets embedded within the PDMS matrix is not present in samples G20_XLR630 and G80_XLR630. Elongated, ellipsoid-like glycerol droplets were formed instead. It is hypothesized that this is an effect of using the thinning agent OS-20, which was rapidly evaporating from the hydrophobic phase of the samples during the cross-linking process. Intuitively, the fluid was evaporating upwards, which led to the orientation of the glycerol droplets in the direction of evaporation. The longest semi-axis of the ellipsoid-like droplets was proved, via SEM analysis, to be parallel to the theoretical direction of evaporation. Despite this anomaly, glycerol droplets were successfully encapsulated within the XLR630 PDMS composition, which was substantiated further in the dielectric spectroscopy results. Adding thinning fluids is a common practice, since it greatly simplifies the processability of high-viscosity polymeric compositions. Importantly, the SEM analysis showed that introducing thinning agents might lead to anisotropy in the resulting composite material, which might also deteriorate the ultimate properties of the material and eventually limit its usefulness. Therefore, a proper balance between evaporation rate and PDMS-curing conditions has to be found for each PDMS system. 

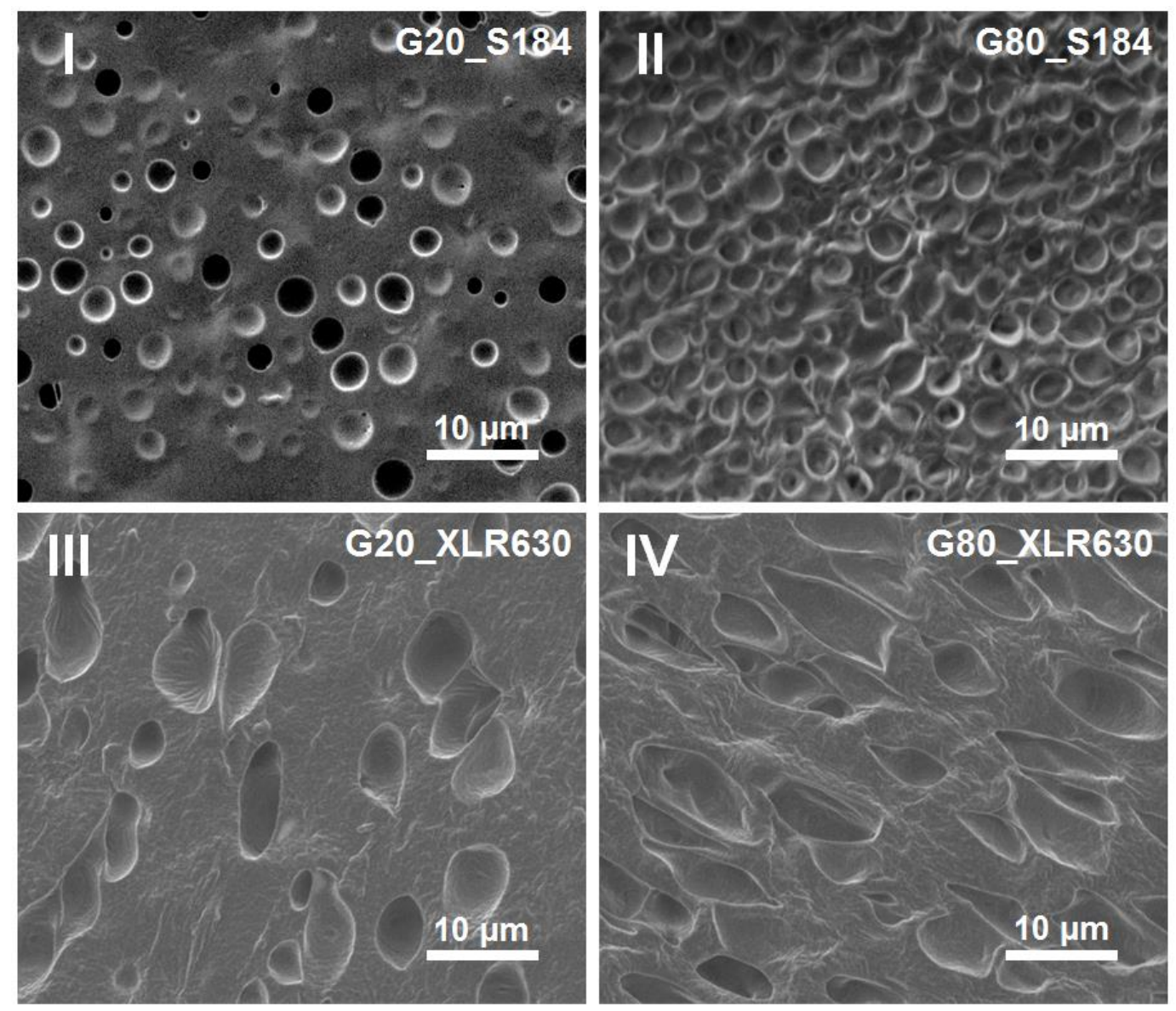

Figure 2. SEM images of cured glycerol-PDMS composite cross-sections.

\subsection{Dielectric spectroscopy}

Broadband dielectric spectroscopy tests were performed in order to determine the dielectric properties of compositions with various amounts of glycerol incorporated. In this study, a two-phase system is described in which a higher-conductivity material is distributed within a lower-conductivity material in the form of discrete droplets. The droplets were expected to act as high-permittivity filler uniformly distributed throughout the dielectric polymer matrix. Since the material is based on a glycerol-PDMS emulsion, it can be assumed that the glycerol droplets are effectively encapsulated within a PDMS insulating layer. Intuitively, this effect should eliminate the probability of the formation of conductive 
paths throughout the material and consequently the conductivity should not be affected by increased amounts of filler. The advantages of filler encapsulation have been thoroughly explained by other groups. ${ }^{[31]}$

As can be seen in Figure 3, a significant increase in dielectric constant is observed with increasing glycerol loading. The permittivity at $1 \mathrm{kHz}$ increases from around 3.3 for neat Sylgard 184 up to around 16 for a sample of G120_S184. Importantly, already a composition with $20 \mathrm{phr}$ of the filler exhibits a significant enhancement of permittivity compared to the neat sample. Consequently, the G20_S184 and G40_S184 samples have dielectric constants of 5.1 and 7.1 , respectively, at $1 \mathrm{kHz}$. It should be noted that all the curves for samples with glycerol incorporated exhibit a characteristic inflection in the frequency region between $10^{4}$ and $10^{6} \mathrm{~Hz}$, which might be evidence of a dipolar relaxation of the high-permittivity filler. The higher the glycerol loading, the steeper the relaxation step and in line with the increasing height of the relaxation step, a characteristic peak in the dielectric loss occurs in the same frequency region. This is an additional indication of the presence of a dipolar relaxation. Importantly, dielectric losses are considered to be exceptionally low for all of the investigated compositions, reaching a maximum of around 2.5 in the higher frequency region. As expected, conductivities are not significantly altered by increasing additions of filler, which justifies the concept of glycerol encapsulation. The conductivity curves also exhibit characteristic slope changes that correspond to the postulated dipole relaxation.

XLR630-based samples also exhibit significant enhancements of their dielectric constants (see Figure 4), which are even more prominent than in the case of S184. A maximum dielectric constant of around 20 was reached with a G120_XLR630 sample. S184 and XLR630 have comparable dielectric constants, and thus the higher values for XLR630-based 
composites are unexpected. The thinning agent is not anticipated to influence the dielectric constant directly, because of its presumed complete evaporation. It can be hypothesized that the difference comes from the shape of the embedded filler, as this might alter interactions between adjacent glycerol droplets.

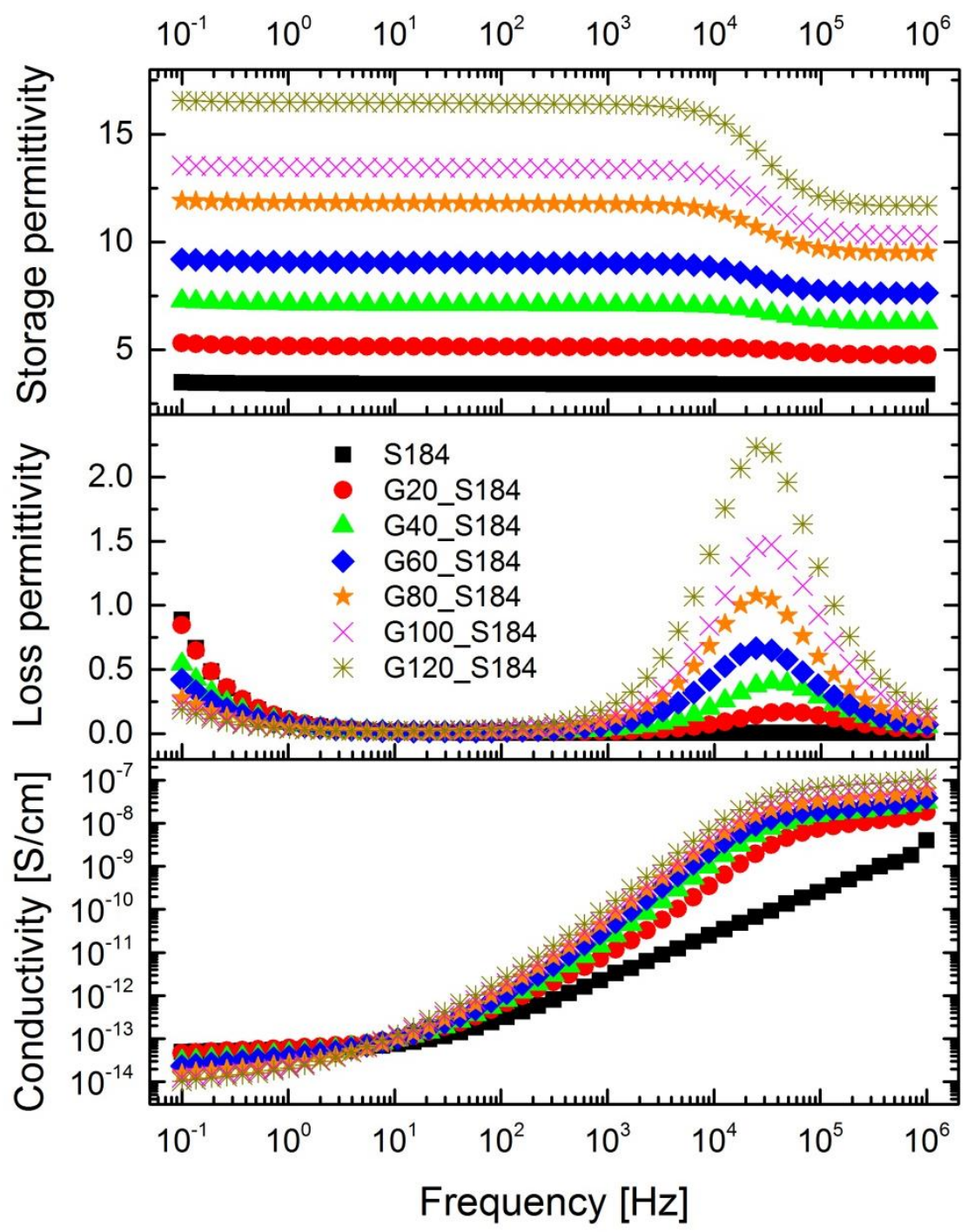

Figure 3. Storage permittivity, loss permittivity and AC conductivity of various glycerolS184 composites at room temperature. 


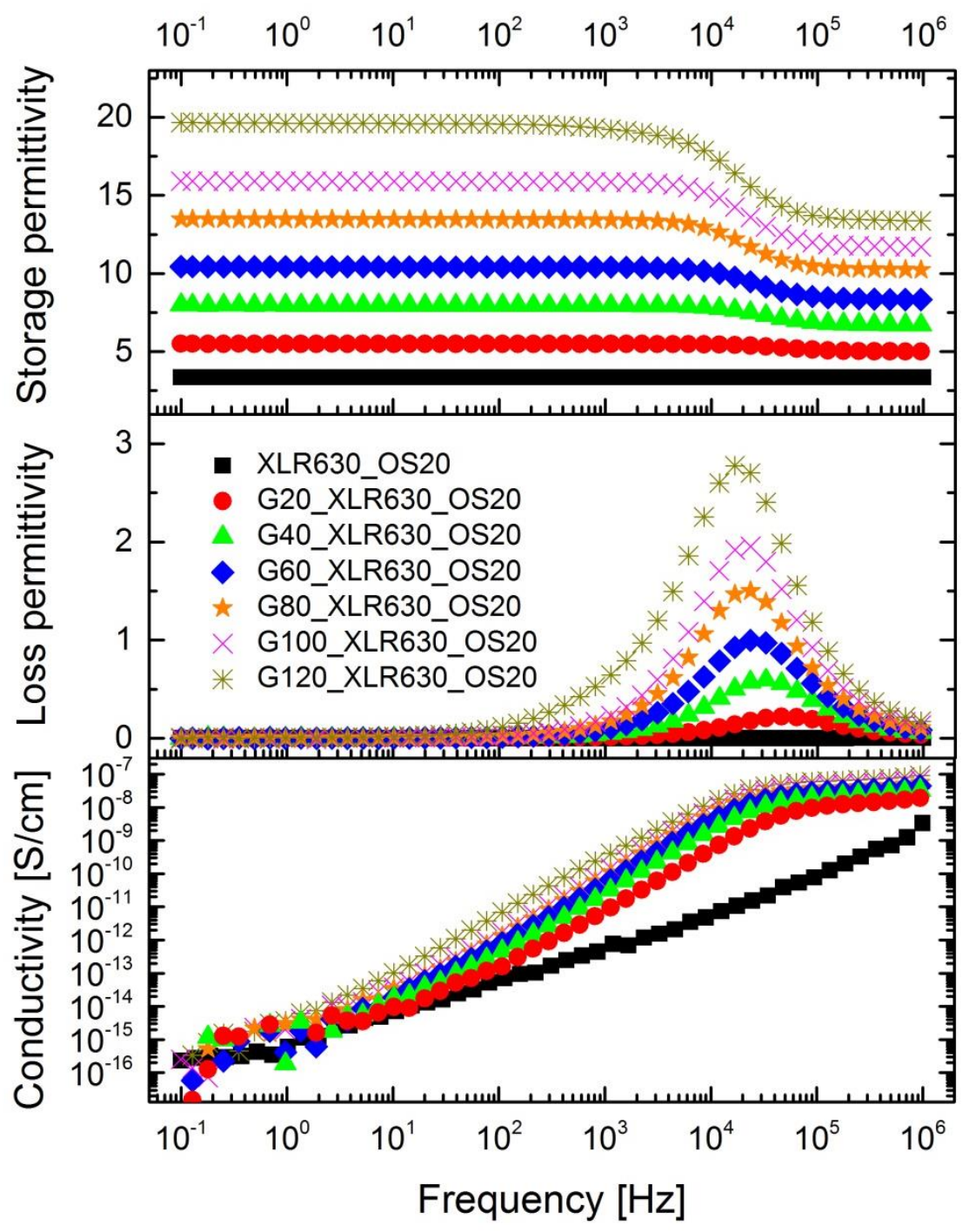

Figure 4. Storage permittivity, loss permittivity and AC conductivity of various glycerolXLR630 composites at room temperature.

The gradual increase in relative permittivity as a function of filler concentration has also been compared to various theoretical models predicting the behavior of composites of lowpermittivity polymers blended with high-permittivity fillers. All models were developed for binary systems, where the inclusions are a spherical. For this reason, only composites based on Sylgard 184 were considered, as the filler can be assumed to be spherical only in this 
case. First, presuming the existence of approximate lower (2) and upper (3) limits for such binary systems, a simple mixing rule is chosen: ${ }^{[32]}$

$$
\begin{aligned}
& \varepsilon_{c, \text { min }}=\frac{\varepsilon_{m} \varepsilon_{f}}{\varepsilon_{m} v_{f}+\varepsilon_{f} v_{m}} \quad \text { and } \\
& \varepsilon_{c, \text { max }}=\varepsilon_{m} v_{m}+\varepsilon_{f} v_{f},
\end{aligned}
$$

where $\varepsilon_{c}, \varepsilon_{m}$ and $\varepsilon_{f}$ are the permittivities of the composite, the matrix and the filler, respectively, while $v_{m}$ and $v_{f}$ are the volume fractions of matrix and filler in the binary composites. The experimental data fit well within the limits of the model. Yet, a more accurate model is necessary in order to follow more precisely the observed trends. More sophisticated predictions can be obtained with one of the earliest mixing formulas, the cocalled Maxwell-Garnett (M\&G) Equation (4) which holds for a relatively broad range of volume fractions: ${ }^{[33,34]}$

$$
\varepsilon_{c}=\varepsilon_{m}\left[1+\frac{3 v_{f}\left(\varepsilon_{f}-\varepsilon_{m}\right)}{v_{m}\left(\varepsilon_{f}-\varepsilon_{m}\right)+3 \varepsilon_{m}}\right]
$$

Corrections to the afore-mentioned equations were introduced by Bruggeman. ${ }^{35}$ His model covers inclusion volume fractions up to 0.5 and is therefore frequently used for describing composites with very high filler loadings. ${ }^{[12]}$ The model may even be applied after the formation of agglomerates, provided the percolation threshold is not exceeded. The equation is usually given in the following form: 


$$
\frac{\varepsilon_{f}-\varepsilon_{c}}{\varepsilon_{c}^{1 / 3}}=\frac{\left(1-v_{f}\right)\left(\varepsilon_{f}-\varepsilon_{m}\right)}{\varepsilon_{c}^{1 / 3}}
$$

Subsequently, the applicability of the well-known Jayasundere-Smith (J\&S) model in the present case was tested. ${ }^{[36,37]}$ In this model, it is assumed that the dielectric constant of the inclusions is substantially higher than that of a dielectric continuum. Additionally, the formula takes into account polarization-based interactions between adjacent particles. Intuitively, the probability of particle-particle interactions is much higher for high filler loadings. Therefore, it is expected that the experimental data will fit better to the predictions from this model, especially at very high inclusion volume fractions. The model is represented by the following equation:

$$
\varepsilon_{c}=\frac{\varepsilon_{m} v_{m}+\varepsilon_{f} v_{f} \frac{3 \varepsilon_{m}}{\left(2 \varepsilon_{m}+\varepsilon_{f}\right)}\left[1+3 v_{f} \frac{\left(\varepsilon_{f}-\varepsilon_{m}\right)}{2 \varepsilon_{m}+\varepsilon_{f}}\right]}{v_{m}+v_{f} \frac{3 \varepsilon_{m}}{\left(2 \varepsilon_{m}+\varepsilon_{f}\right)}\left[1+3 v_{f} \frac{\left(\varepsilon_{f}-\varepsilon_{m}\right)}{2 \varepsilon_{m}+\varepsilon_{f}}\right]}
$$

Last but not least, a model proposed by Poon and Shin (P\&S) (Equation (7)) is considered. The formula was developed in order to find a model that may fit as broad a range of systems as possible. ${ }^{38}$ The formula is not limited to the case of $\varepsilon_{f} \gg \varepsilon_{m}$ (as with of Equation (6), Jayasundere-Smith) and covers even situations where $\varepsilon_{f}<\varepsilon_{m}$. Furthermore, the simplicity of Equation (7) is one of its strengths. As the authors argue, more complicated non-linear equations (such as Equation (5), Bruggeman) have limited use in situations where a simpler formula may be employed: 


$$
\varepsilon_{c}=\varepsilon_{m}+\frac{v_{f}\left(\varepsilon_{f}-\varepsilon_{m}\right)}{v_{f}+v_{m} \frac{\varepsilon_{f}+2 \varepsilon_{m}-v_{f}\left(\varepsilon_{f}-\varepsilon_{m}\right)}{3 \varepsilon_{m}}}
$$

Experimental values of the storage permittivity at a frequency of $1 \mathrm{kHz}$ were used for comparisons between the various models. Between $0.1 \mathrm{~Hz}$ and $10 \mathrm{kHz}$, the curves exhibit a plateau so that the analysis may be assumed to be valid for a broader frequency range. As can be seen in Figure 5, the model proposed by Jayasundere and Smith fits the experimental data in the best way. The theoretical estimate fits the data from lower glycerol loadings up to loadings of around $100 \mathrm{phr}$, and the value at $120 \mathrm{phr}$ of glycerol deviates only slightly from the model curve. As discussed before, the material becomes more conductive at very high inclusion loadings (>120 phr). Here, the deviation is not significant, but might be attributed to the formation of a continuous glycerol phase and of conductive pathways. All models have been developed for 0-3 composites (spherical filler particles embedded in a continuous matrix), and thus the validity of the equations is restricted to filler loadings below 120 phr. 


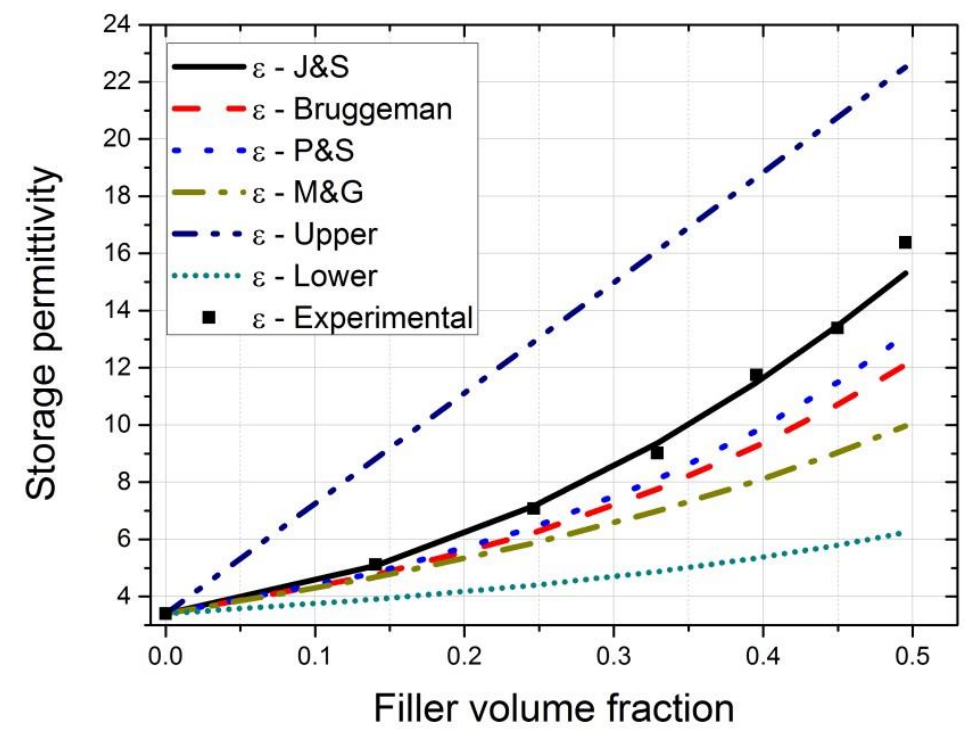

Figure 5. Experimental data and theoretical estimate of the relative permittivity at $1 \mathrm{kHz}$ as a function of the glycerol loading in the composites.

As can be seen in Figure 3 and 4, the conductivities in the lower frequency region of the glycerol-PDMS composites do not change significantly with increasing glycerol loadings. However, the SEM analysis showed that the insulating layers between the glycerol droplets become thinner for increasing amounts of filler, as expected. Therefore, the chances for charge migration between the droplets become higher. Although higher conductivities are not to be expected at $1 \mathrm{~V}$, tests at higher voltages were performed in order to validate the usefulness of the material for high-voltage applications. Several samples, of G100_S184 and G100_XLR630_OS20 were subjected to increasing AC voltages, and the results are presented in Figure 6. The conductivity of G100_S184 increases significantly with increasing voltage, while the conductivity of G100_XLR630_OS20 remains almost unaffected. The conductivity of unmodified XLR630, tested at $1 \mathrm{~V}$, is lower than that of S184 under the same conditions (cf. Figure 3 and 4). As the amount of inclusions is exactly the same in both samples, it can be concluded that the type of matrix is essential for the 
conductivity of glycerol-PDMS composites. Intuitively, materials with higher resistivity (resistivity is the reciprocal of the conductivity) will insulate the glycerol droplets more efficiently and block charge transfer between adjacent spheres. Ultimately, the probability of forming conductive paths will be considerably restricted in a dielectric matrix with higher resistivity.

As discussed, the thinner the insulating layer between the inclusion droplets, the higher the conductivity. This is also demonstrated by the results presented in Figure 7, in which the conductivities of samples G80_S184 and G100_S184 are compared. As can be observed, the conductivity of the sample with $80 \mathrm{phr}$ is not influenced by a voltage increase from $1 \mathrm{~V}$ to $350 \mathrm{~V}$, whereas for the sample with $100 \mathrm{phr}$, the conductivity drastically increases at $350 \mathrm{~V}$. This suggests the existence of a required minimum spacing for separating and insulating adjacent glycerol droplets from each other. It is believed that the threshold for the shell thickness would be lower in materials with higher electrical resistivity.
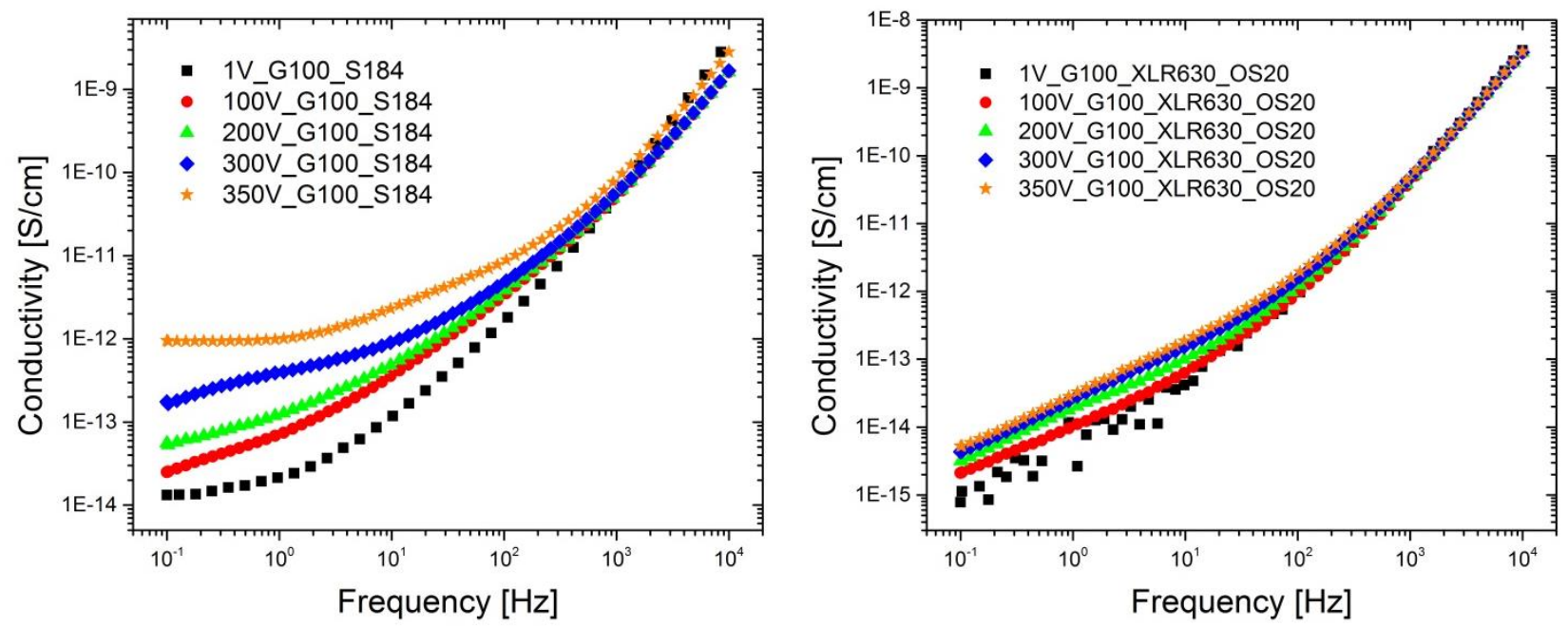

Figure 6. Conductivities of composites based on S184 (left) and XLR630 (right), containing $100 \mathrm{phr}$ of glycerol for various AC voltages at room temperature. 


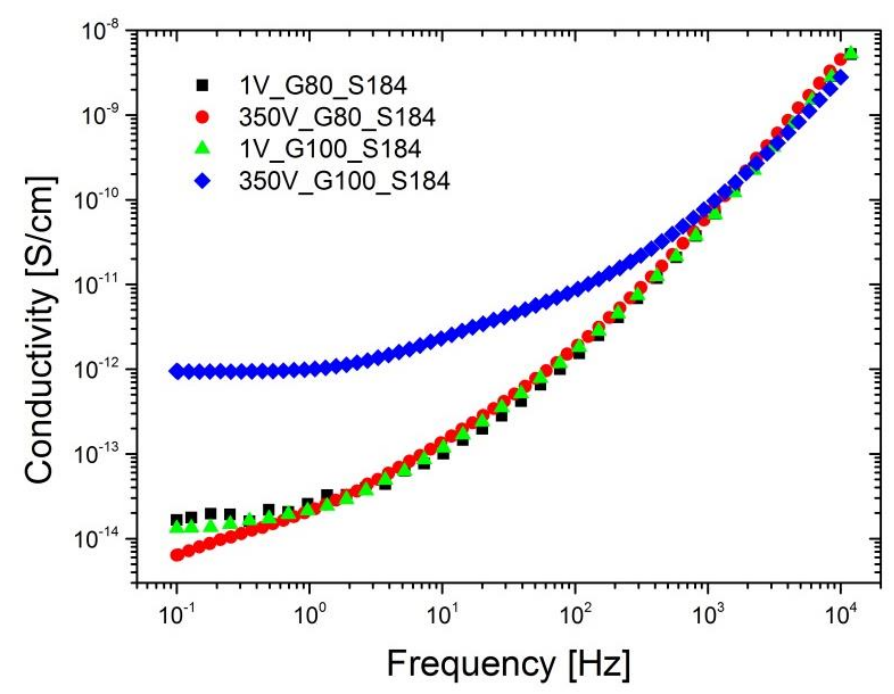

Figure 7. Conductivity of various glycerol-S184 composites for $1 \mathrm{~V}$ and $350 \mathrm{~V}$, respectively, at room temperature.

\subsection{Modification of droplet size and its influence on the dielectric constant of glycerol-PDMS composites}

In the previous section, well-known theoretical models for predicting the dielectric constants of composites were evaluated. Additional reports that emphasize the necessity of considering a polymer-filler interphase as an additional factor influencing the final dielectric constant of a material can be found in the literature. ${ }^{[39,40]}$ Furthermore, Vo and Shi discuss the presence of a molecular polarizability at the filler-polymer interphase, which might also influence the resulting permittivity. ${ }^{[41]}$ As the filler-polymer interphase increases in line with a decrease in filler size at a given loading, it is expected that a change in relative permittivity will be observed.

Various surfactants in different amounts were added to the G10_S184 pre-elastomer composition, and their influence on droplet diameters was investigated. First, $1 \mathrm{~mm}$-thick samples of PDMS, containing 10 phr of glycerol with the addition of Tween 20 (a glycerol- 
miscible, polysorbate-based surfactant), were prepared according to the standard procedure used in this study. SEM images of samples with 0.1 and 5 parts by weight of the surfactant are shown in Figure 8a. The data presented in Figure $8 \mathbf{b}$ indicate that an increasing amount of surfactant in a composition leads to the formation of smaller glycerol droplets, along with a narrower size distribution. The average droplet diameter decreases from $2.3 \mu \mathrm{m}$ for the non-surfactant sample to $0.8 \mu \mathrm{m}$ for the highest Tween 20 loading investigated here. The dielectric constant increased from 4.2 for pure G10_S184 to 5.0 with the addition of 5 phr of Tween 20. Nevertheless, the steepest increase was observed for smaller amounts of Tween 20, which correlates well with the stronger decrease in droplet diameter at lower surfactant loadings. Remarkably, the dielectric constant of the sample G20_S184 was found to be at 5.1, whereas the sample G10_S184 with the addition of only 3 phr of Tween 20 reached a dielectric constant of 4.9. This implies that a strong dependence exists between dielectric constant and filler size, i.e. that the space-charge motion within the composite is more remarkable in the S18-composites.

A significant decrease in the average droplet diameter was observed also after incorporating two PEG-PDMS surfactants (block-copolymer-based surfactants with molecular weights of $400 \mathrm{~g} / \mathrm{mol}$ and $3000 \mathrm{~g} / \mathrm{mol}$, respectively, with comparable ratios between the PEG and the PDMS blocks) into the G10_S184 composition. The influence on the droplet diameters was similar to the effects of Tween 20; however, no clear increase in the dielectric constant was observed on these samples.

The aim of this approach was to investigate how the volume of the filler-polymer interphase influences the dielectric constant of composites. The contradictory results indicate that the properties vary with the constituents that form the interphase, suggesting a high complexity of the processes that occur in the interphase. It is therefore necessary to consider each system separately. 
It has to be stressed that the diameters obtained from evaluating the cross-sections do not correspond to the actual droplet diameters, as it is not possible to prepare sample cross-sections by cutting each inclusion droplet through its respective center. Nevertheless, for a comparative study, the present approach is considered to be sufficiently accurate.
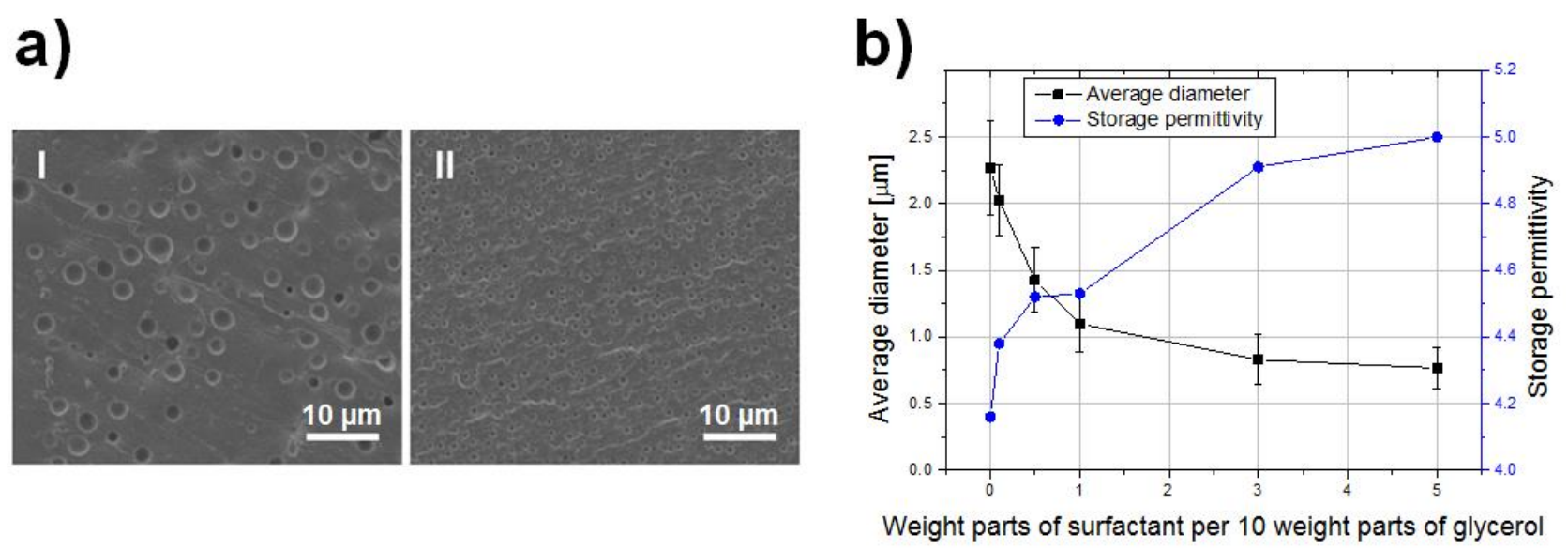

Figure 8. Effect of surfactant on droplet size. a) SEM images of cured samples G10_S184 with 0.1 (I) and 5 (II) phr of Tween 20. The scale bars in both images correspond to $10 \mu \mathrm{m}$. b) Average glycerol droplet diameter and storage permittivity at $1 \mathrm{kHz}$ as a function of surfactant content in various Tween 20-glycerol-PDMS composite.

\section{Conclusions}

The use of glycerol-PDMS green elastomers and their potential applicability as dielectric elastomers was proposed, investigated and discussed. The novel class of materials has been proven to exhibit high dielectric constants strongly depending on the glycerol loading. Furthermore, the glycerol-PDMS composites were shown to exhibit very low dielectric losses. The composites were assessed by means of some of the most popular theoretical 
models predicting changes of relative permittivities as a function of filler content. Results show that the formula suggested by Jayasundere and Smith fits the experimental data best. The J\&S theory takes into account interactions between adjacent filler particles, which intuitively intensify at higher glycerol loadings. This factor is considered to be the main advantage of the model.

The high-voltage dielectric spectroscopy measurements indicate that the conductivity tends to increase with increasing voltages and filler loadings. Nonetheless, the experimental results indirectly also show that this problem can be overcome by using the best possible dielectric material as a matrix for composites. It is believed that a highly resistive dielectric matrix would be capable of preventing charge transfer between adjacent glycerol droplets. This is especially important at high filler loadings, where the distances between the droplets become very small.

The novel elastomer material exhibits several interesting features from the perspective of dielectric-elastomer transducer development with a very high dielectric constant and a tunable elastic modulus being the two most important ones. The simplicity of preparation and the variety of potential candidates for the matrix and the inclusions are other key aspects of the new concept.

Acknowledgements

The authors acknowledge funding from Innovationsfonden Denmark and from the DFG and the EU for equipment.

Keywords: 
dielectric elastomer, silicone, glycerol, filler, hybrid elastomers

1 W. C. Röntgen, Ann. Phys. Chem, 1880, 11, 771-786.

2 C. Keplinger, M. Kaltenbrunner, N. Arnold and S. Bauer, Proc. Natl. Acad. Sci. U. S. A., 2010, 107, 4505-4510.

3 R. Pelrine, R. Kornbluh, J. Joseph, R. Heydt, Q. Pei and S. Chiba, Mater. Sci. Eng. C, 2000, $11,89-100$.

4 M. Zhenyi, J. I. Scheinbeim, J. W. Lee and B. A. Newman, J. Polym. Sci. Part B Polym. Phys., 1994, 32, 2721-2731.

$5 \quad$ Y. M. Shkel and D. J. Klingenberg, J. Appl. Phys., 1996, 80, 4566-4572.

6 R. Pelrine, Science, 2000, 287, 836-839.

7 R. Kochetov, I. A. Tsekmes and P. H. F. Morshuis, Smart Mater. Struct., 2015, 24, 075019.

8 G. Gallone, F. Galantini and F. Carpi, Polym. Int., 2010, 59, 400-406.

9 K. Goswami, F. Galantini, P. Mazurek, A. E. Daugaard, G. Gallone and A. L. Skov, Smart Mater. Struct., 2013, 22, 115011.

10 J. Huang, S. Shian, Z. Suo and D. R. Clarke, Adv. Funct. Mater., 2013, 23, 5056-5061.

11 T. G. McKay, S. Rosset, I. A. Anderson and H. Shea, Smart Mater. Struct., 2015, 24, 015014.

12 G. Gallone, F. Carpi, D. De Rossi, G. Levita and A. Marchetti, Mater. Sci. Eng. C, 2007, 27, $110-116$.

13 S. Vudayagiri, S. Zakaria, L. Yu, S. S. Hassouneh, M. Benslimane and A. L. Skov, Smart Mater. Struct., 2014, 23, 105017. 
14 A. E. Daugaard, S. S. Hassouneh, M. Kostrzewska, A. G. Bejenariu and A. L. Skov, Proc. SPIE, 2013, 8687, 868729-1.

15 K. Goswami, A. E. Daugaard and A. L. Skov, RSC Adv., 2015, 5, 12792-12799.

16 A. Javadi, Y. Xiao, W. Xu and S. Gong, J. Mater. Chem., 2012, 22, 830-834.

17 F. Carpi, G. Gallone, F. Galantini and D. De Rossi, Adv. Funct. Mater., 2008, 18, 235-241.

18 F. B. Madsen, A. E. Daugaard, S. Hvilsted, M. Y. Benslimane and A. L. Skov, Smart Mater. Struct., 2013, 22, 104002.

19 F. B. Madsen, L. Yu, A. E. Daugaard, S. Hvilsted and A. L. Skov, RSC Adv., 2015, 5, $10254-10259$.

20 F. B. Madsen, A. E. Daugaard, S. Hvilsted and A. L. Skov, Macromol. Rapid Commun., 2016, 37, 378-413.

21 P. Mazurek, A. E. Daugaard, M. Skolimowski, S. Hvilsted and A. L. Skov, RSC Adv., 2015, $5,15379-15386$.

22 P. Mazurek, S. Hvilsted and A. L. Skov, Proc. SPIE, 2014, 9056, 90562T-1.

23 Y. Zhang, M. Ishida, Y. Kazoe, Y. Sato and N. Miki, IEEJ Trans. Electr. Electron. Eng., 2009, 4, 442-449.

24 E. Bormashenko, Soft Matter, 2012, 8, 11018-11021.

25 E. Bormashenko, R. Pogreb, T. Stein, G. Whyman, M. Schiffer and D. Aurbach, J. Adhes. Sci. Technol., 2011, 25, 1371-1377.

26 Y. Xue, H. Wang, Y. Zhao, L. Dai, L. Feng, X. Wang and T. Lin, Adv. Mater., 2010, 22, $4814-4818$. 
27 Y. Zhao, Z. Xu, M. Parhizkar, J. Fang, X. Wang and T. Lin, Microfluid. Nanofluidics, 2012, $13,555-564$.

28 P. Aussillous and D. Quéré, Nature, 2001, 411, 924-927.

29 E. Bormashenko, R. Pogreb, R. Balter, O. Gendelman and D. Aurbach, Appl. Phys. Lett., 2012, 100, 151601.

30 P. Mazurek, S. Hvilsted and A. L. Skov, Polymer, 2016, 87, 1-7.

31 W. Hu, S. N. Zhang, X. Niu, C. Liu and Q. Pei, J. Mater. Chem. C, 2014, 2, 1658-1666.

32 F. Carpi, D. De Rossi, R. Kornbluh, R. Pelrine and P. Sommer-Larsen, Dielectric Elastomers as Electromechanical Transducers, Elsevier, Amsterdam, 2008, p. 55.

33 J. C. M. Garnett, Philos. Trans. R. Soc. A Math. Phys. Eng. Sci., 1906, 205, 237-288.

34 E. Tuncer, S. M. Gubański and B. Nettelblad, J. Appl. Phys., 2001, 89, 8092-8100.

35 S. O. Nelson and T. S. You, J. Phys. D. Appl. Phys., 1990, 23, 346-353.

36 N. Jayasundere and B. V. Smith, J. Appl. Phys., 1993, 73, 2462-2466.

37 N. Jayasundere, B. V. Smith and J. R. Dunn, J. Appl. Phys., 1994, 76, 2993-2998.

38 Y. M. Poon and F. G. Shin, J. Mater. Sci., 2004, 39, 1277-1281.

39 M. Ezzat, N. A. Sabiha and M. Izzularab, Appl. Nanosci., 2013, 4, 331-338.

40 M. G. Todd and F. G. Shi, J. Appl. Phys., 2003, 94, 4551-4557.

41 H. T. Vo and F. G. Shi, Microelectronics J., 2002, 33, 409-415. 


\section{The table of content}

A novel two-phase glycerol-silicone elastomer was created. An easy preparation procedure, great versatility of the concept and multiple inspiring properties are main advantages of this new class of stretchable materials. We hereby present a pioneering attempt of using glycerol-silicone composites as high-dielectric constant and low Young's modulus membranes for dielectric elastomer actuators.
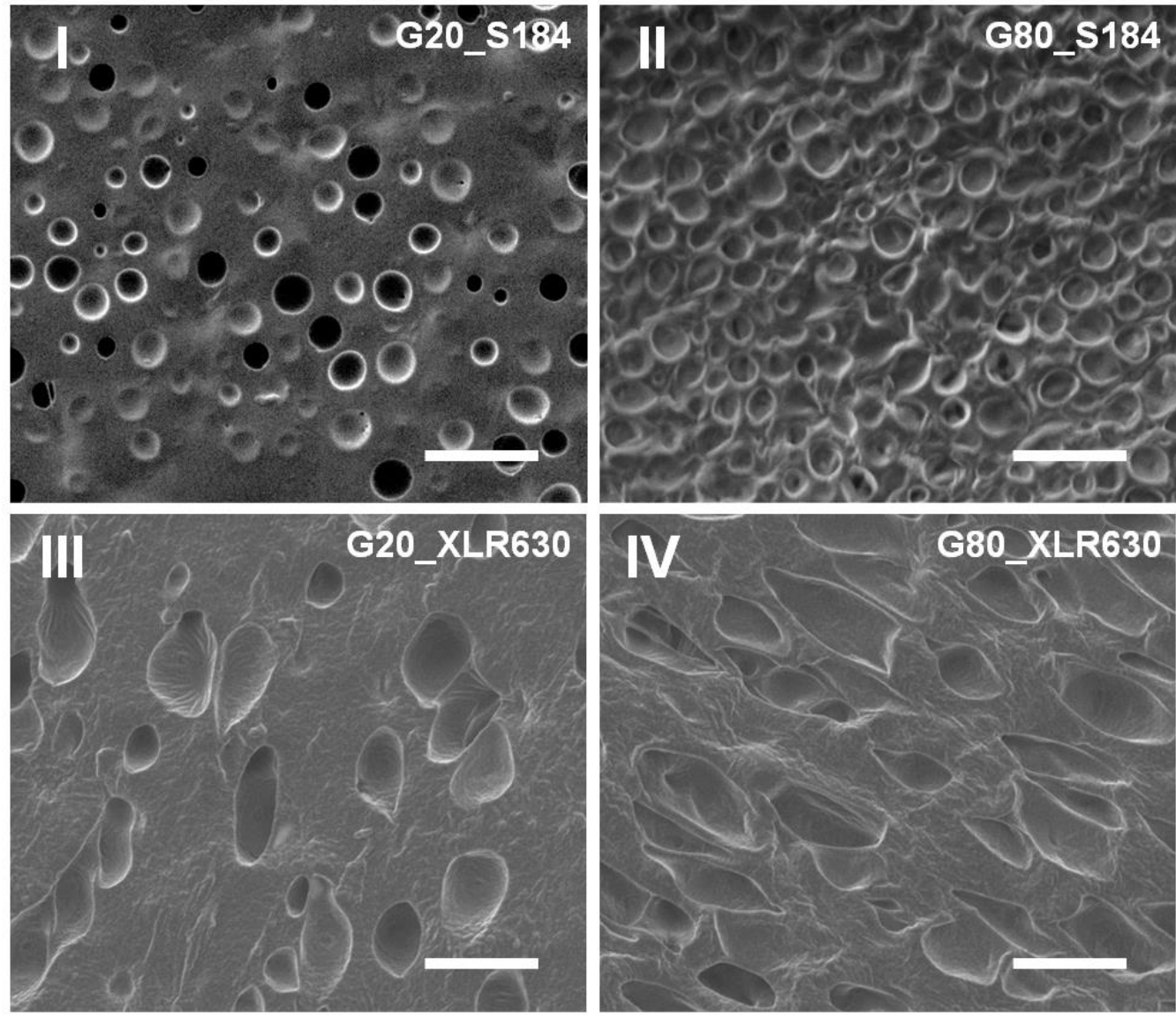